\title{
Concordância entre dois critérios de classificação da atividade física e fatores associados em adolescentes
}

\section{Agreement between two criteria of physical activity classification and associated factors in adolescents}

\author{
André de Araújo Pinto', Gaia Salvador Claumann', Rita Maria dos Santos Puga Barbosa², Markus Vinicius Nahas³, Hector \\ Cris Colares de Angelo', Andreia Pelegrini ${ }^{1}$
}

\section{Resumo}

O objetivo deste estudo foi analisar a concordância entre dois critérios de classificação da atividade física (AF) e os respectivos fatores associados. Os participantes foram 2.517 adolescentes amazonenses (14 a 19 anos de idade). O nível de AF e as variáveis independentes (sexo, faixa etária, ano escolar, turno de estudo, renda familiar, escolaridade materna e situação ocupacional) foram coletados por meio de questionário. O coeficiente kappa e a regressão logística binária foram empregados, respectivamente, para analisar a concordância entre as prevalências e os fatores associados aos dois critérios de classificação da AF (300 e 420 minutos/semana). Verificou-se moderada concordância entre os critérios (kappa $=0,58 ; \mathrm{p}<0,001$ ) para classificação do nível de AF, com diferença de 8,5 pontos percentuais entre as prevalências, sendo que para os 300 e 420 minutos/semana foram classificados como fisicamente ativos 15,5\% (IC95\% = $14,2-16,7)$ e $7,0 \%($ IC95\% $=6,0-7,0)$ dos participantes, respectivamente. Os adolescentes do sexo masculino e os que não trabalhavam tiveram mais chances de serem fisicamente ativos em ambos os critérios. Os adolescentes mais novos (14 a 16 anos) tiveram mais chances de serem fisicamente ativos no critério de 300 minutos/semana. O ano escolar, turno de estudo, renda familiar e escolaridade materna não se associaram aos critérios utilizados. A concordância entre os dois critérios de classificação do nível de $\mathrm{AF}$ foi moderada e os fatores associados foram semelhantes em ambos os critérios, com exceção da faixa etária.

\section{Palavras-chave}

Atividade física; Adolescente; Epidemiologia; Saúde Pública.

\begin{abstract}
This study aimed to analyze the agreement between two criteria to classify physical activity (PA), and the respective associated factors. Participants were 2.517 adolescents (aged 14 to 19) from Amazonas. The PA level and the independent variables (sex, age group, school year, school shift, family income, maternal educational level and work) were collected by means of a questionnaire. The kappa coefficient and binary logistic regression were used to analyze the agreement between the prevalence and the associated factors to both criteria (300 and 420 minutes/week of PA), respectively. We found a moderate agreement between the two criteria to classify $P A$ level (kappa $=0.58)$, with a difference of 8.5 percentage points between the prevalence, considering that for 300 and 420 minutes/ week, $15.5 \%(95 \% C I=14.2-16.7)$ and $7.0 \%(95 \% C I=6.0-7.0)$ of the participants were classified as physically active, respectively. Male adolescents and those who did not work were more likely to be physically active in both criteria, compared to their peers. Younger adolescents (14 to 16 years) were more likely to be physically active in the criterion of 300 minutes/week. School year, school shift, family income and maternal educational level were not associated to the used criteria. In conclusion, the agreement between two criteria to classify PA level was moderated and the associated factors to sufficient PA were similar in both criteria with an exception for age group.
\end{abstract}

\section{Keywords}

Adolescent; Epidemiology; Physical Activity; Public Health.
1 Universidade do Estado de Santa Catarina, Centro de Ciências da Saúde e do Esporte, Programa de Pós-graduação em Ciências do Movimento Humano, Florianópolis, Santa Catarina, Brasil.

2 Universidade Federal do Amazonas, Faculdade de Educação Física e Fisioterapia, Manaus, Amazonas, Brasil.

3 Universidade Federal de Santa Catarina, Departamento de Educação Física, Florianópolis, Santa Catarina, Brasil.

\section{Introdução}

A prática regular de atividade física $(\mathrm{AF})$ tem sido reconhecida como um comportamento que contribui para a prevenção e o tratamento de doenças, manutenção da saúde e melhora da qualidade de vida ${ }^{1}$. Porém, para que tais benefícios sejam obtidos, é ne- 
cessário o engajamento em atividades físicas levando em consideração frequência e intensidade adequadas. Especificamente para adolescentes, as recomendações mundiais sugerem pelo menos 60 minutos diários de AF de intensidade moderada e vigorosa (AFMV), sendo que maiores benefícios podem ser alcançados com o aumento da prática de $\mathrm{AF}^{2}$. Apesar de existirem essas orientações, tem crescido a discussão entre os pesquisadores sobre as quantidades mínimas necessárias de AF nessa fase da vida em particular ${ }^{3}$.

Estudos epidemiológicos conduzidos com adolescentes de diferentes regiões brasileiras, com o objetivo de estimar a prevalência de AFMV suficiente, têm utilizado principalmente o critério de pelo menos $300^{4-10}$ e também de $420^{5}$ minutos/ semana para classificar os níveis de AF. Dentre os estudos que empregaram 300 minutos/semana de AFMV, as prevalências de AFMV suficiente variaram de 7,9\% ${ }^{4}$ a $59,1 \%{ }^{10}$. Apesar da gravidade desses achados, pressupõe-se que essas prevalências podem ter sido superestimadas visto que não foram considerados os 420 minutos/semana mínimos de AFMV propostos pela Organização Mundial de Saúde². Assim, pesquisadores tem recomendado ao menos a padronização do critério de classificação de $\mathrm{AF}^{3,11-13}$, uma vez que são inúmeros os instrumentos utilizados para a sua mensuração ${ }^{11}$, e os procedimentos que incluem desde a triagem amostral à análise dos resultados ${ }^{3}$.

Inquéritos com o foco na $\mathrm{AF}$ e saúde conduzidos em adolescentes brasileiros foram realizados, principalmente, nas regiões Sul e Sudeste, observando-se a escassez de estudos sobre essa temática na região Norte ${ }^{11}$. Essa situação é justificada, possivelmente, pela inexistência de programas de Pós-graduação em Educação Física nessa região e, especialmente no Amazonas, o que limita o desenvolvimento de pesquisas nessa área de conhecimento. Além disso, a região Norte, de forma geral, apesar de ser considerada a maior região brasileira em termos territoriais, possui municípios muito distantes uns dos outros e a ligação entre eles é, na maioria das vezes, feita por meio dos extensos rios, sendo mais um fator de dificuldade para o desenvolvimento de pesquisas epidemiológicas.

Para além da necessidade de pesquisas sobre AF na região Norte e apesar de haver diretrizes mais recentes sobre a prática de $\mathrm{AF}^{2}$, observa-se, na literatura pesquisada, que o emprego do critério de 420 minutos/semana ainda é pouco utilizado. Ressalta-se também que a ausência de um consenso sobre a quantidade ideal de AF pode refletir em dificuldades no desenvolvimento de políticas públicas que promovam sua prática regular ${ }^{14}$.

Diante do exposto, torna-se relevante investigar as possíveis diferenças entre os critérios para classificação dos níveis de AF, no sentido de compreender se a utilização de um determinado critério em detrimento a outro resulta em prevalências semelhantes. Ademais, não se têm informações consistentes na literatura de que os possíveis fatores associados à $\mathrm{AF}$ em adolescentes sejam semelhantes ou diferentes conforme o critério utilizado. Diante do exposto, pressupõe-se que a utilização de diferentes critérios de classificação poderá resultar em estimativas de prevalências de AF divergentes e, consequentemente, influenciar na identificação de seus fatores associados. Para testar essas hipóteses, o objetivo deste estudo foi analisar a concordância entre dois critérios de classificação da atividade física (AF) e os respectivos fatores associados.

\section{Método}

Estudo epidemiológico, de delineamento transversal, realizado com adolescentes da rede pública estadual de ensino do Amazonas no ano de 2011, vinculado ao 
macroprojeto "Estilo de Vida e Indicadores de Saúde de Escolares do Ensino Médio do Amazonas". O presente estudo é resultado de uma parceria entre a Universidade Federal do Amazonas (UFAM), Universidade do Estado de Santa Catarina (UDESC) e Universidade Federal de Santa Catarina (UFSC). Esta pesquisa foi aprovada pelo Comitê de Ética em Pesquisa com Seres Humanos da UFAM (registro CAAE $n^{\circ}$ 0302.0.115.000-11).

A população alvo do estudo foi de adolescentes, com idades de 14 a 19 anos, regularmente matriculados no ensino médio em cinco cidades do Amazonas (Itacoatiara, Manaus, Parintins, Presidente Figueiredo e São Gabriel da Cachoeira). Segundo informações da Secretaria de Estado de Educação do Amazonas, encontravam-se matriculados, no ano de realização da pesquisa, nas cinco cidades, 88.562 adolescentes, sendo 4.164 em Itacoatiara, 78.498 em Manaus, 4.863 em Parintins, 249 em Presidente Figueiredo e 768 em São Gabriel da Cachoeira. Estas cidades foram selecionadas de maneira intencional pois o estado do Amazonas apresenta, em sua maioria, cidades ribeirinhas, o que dificulta o acesso e a coleta das informações.

Os procedimentos sugeridos por Luiz e Magnanini ${ }^{15}$ foram utilizados para determinar o tamanho necessário da amostra, considerando-se o número de alunos matriculados em cada cidade. Para tanto, considerou-se nível de confiança de 95\%, prevalência de desfecho de $50 \%$, erro amostral de cinco pontos percentuais e efeito de delineamento de 1,5. Acrescentou-se $10 \%$ a amostra estimada para cada cidade, com intuito de amenizar possíveis perdas e recusas. A partir desses parâmetros, estimou-se uma amostra total de 2.485 adolescentes (Itacoatiara $=580$; Manaus =631; Parintins $=587$; Presidente Figueiredo $=264 ;$ e São Gabriel da Cachoeira $=$ 423). Em virtude do processo de amostragem por conglomerado de turmas, em que os adolescentes presentes em sala de aula, no momento da coleta foram convidados a fazer parte do estudo, o número de participantes foi de 3.267 (Itacoatiara = 580; Manaus $=1.413 ;$ Parintins $=575 ;$ Presidente Figueiredo $=249 ;$ e São Gabriel da Cachoeira $=450$ ).

O poder do teste para as análises de associação foi calculado a posteriori. Para tal considerou-se a amostra de 2.517 adolescentes, prevalência de AFMV esperada de $12,8 \%$ e razão de prevalência de $0,34^{5}$. Adotando-se o nível de significância de $5 \%$ e poder do teste de $90 \%$ a amostra mínima necessária para a identificação de diferenças significativas na regressão logística foi de 458 adolescentes.

A seleção da amostra ocorreu de maneira distinta nas cinco cidades. Em Manaus, a amostra foi selecionada a partir de três estágios: a) proporcional por distritos de educação ( $n=6$ ), em que todas as escolas foram consideradas elegíveis; b) estratificado por escolas públicas estaduais, considerando-se o número total de alunos (grande porte: 500 alunos ou mais; médio porte: de 201 a 499 alunos; e pequeno porte: até 200 alunos); e c) conglomerado de turmas sorteadas, ano escolar e turno, em que todos os alunos presentes em sala, no momento da coleta foram convidados a participar da pesquisa. Nas cidades Itacoatiara, Parintins e São Gabriel da Cachoeira, em função do baixo número de escolas, foram empregados os estágios "b" e "c". Em Presidente Figueiredo, foi realizado um censo nas duas únicas escolas estaduais do município.

\section{Procedimentos e instrumentos}

Após a autorização da Secretaria de Estado de Educação, as escolas foram contatadas para apresentação do projeto, seus objetivos e importância. Uma equipe de pesquisadores, previamente treinados, foi responsável pela condução da coleta de dados que ocorreu em dias e horários agendados com a escola, durante as aulas de 
Educação Física, em sala de aula. A coleta de dados ocorreu durante todo o ano de 2011, no período letivo.

Os adolescentes receberam informações sobre a importância da pesquisa e tiveram conhecimento dos objetivos que motivaram a condução da mesma. Foram incluídos apenas aqueles que se dispuseram a participar de maneira voluntária, assinaram o Termo de Assentimento e apresentaram o Termo de Consentimento Livre e Esclarecido assinado por algum responsável (para aqueles com idade < 18 anos) ou por eles mesmos (idade $\geq 18$ anos).

As informações dos adolescentes foram obtidas por meio do autorrelato, mediante a aplicação do questionário COMPAC (Comportamento dos adolescentes Catarinenses $)^{16}$, o qual foi traduzido e adaptado para o português brasileiro, tendo sua reprodutibilidade testada em 107 adolescentes (15 a 19 anos de idade) da cidade de Florianópolis, com proporção de concordância de 92,8\% e índice kappa igual a 0,81 para AFMV (<300 minutos/semana $v s . \geq 300$ minutos semana) (dados não publicados).

As variáveis coletadas foram sexo (masculino, feminino), faixa etária (14-16, 17-19 anos), ano escolar (primeiro, segundo, terceiro) e turno de estudo (diurno, noturno). Foram coletadas, ainda, informações sobre a renda familiar, com base no salário mínimo em vigor no ano da pesquisa (até dois salários, de três a cinco salários e seis salários ou mais), nível de escolaridade da mãe (< oito anos de estudo, $\geq$ oito anos de estudo) e situação ocupacional (trabalha, não trabalha).

A AF habitual foi estimada a partir de duas questões, uma que diz respeito à frequência: "Durante uma semana normal (típica), em quantos dias você pratica atividades físicas moderadas a vigorosas (atividades físicas no lazer, no trabalho e deslocamento)?” Os adolescentes tinham oito alternativas como possíveis repostas que variavam de zero a sete dias; e a outra com referência à duração em minutos: "Durante uma semana normal (típica), quanto tempo você pratica atividades físicas moderadas a vigorosas (atividades físicas no lazer, no trabalho e deslocamento)?” As alternativas eram: a) não pratico; b) menos de 30 minutos por dia; c) de 30 a 59 minutos por dia; e d) $60 \mathrm{mi}$ nutos ou mais. Os critérios empregados para classificar o nível de AF dos adolescentes foram de pelos menos 60 minutos diários de AFMV (420 minutos/semana) e 60 minutos de AFMV por pelo menos cinco dias².

\section{Análise estatística}

Os dados foram analisados por meio de estatística descritiva (distribuição de frequências absoluta e relativa) e inferencial. A concordância entre as prevalências da AFMV suficiente na amostra total foi avaliada utilizando-se o Índice de Concordância Kappa de Cohen ${ }^{17}$, sendo a força de concordância classificada conforme sugestão de Landis e Koch ${ }^{18}$ : sem concordância $(<0,00)$, desprezível $(0,00-0,20)$, leve $(0,21$ - 0,40), moderada $(0,41-0,60)$, alta $(0,61-0,80)$ e concordância perfeita $(0,81-1,00)$. Diferenças nas prevalências de AFMV suficiente entre as categorias das variáveis independentes foram analisadas por meio da sobreposição dos intervalos de confiança de $95 \%$. Foram realizadas análises bruta e ajustada da Regressão Logística Binária para verificar a associação da AFMV (classificada de acordo com os critérios de pelo menos 300 e 420 minutos/semana), adotando-se como categoria de referência os insuficientemente ativos, com as variáveis independentes (sexo, faixa etária, turno, ano escolar, turno, renda familiar, escolaridade materna e situação ocupacional). $\mathrm{Na}$ análise ajustada, todas as variáveis foram incluídas simultaneamente no modelo, por meio do método enter, independentemente do valor de $\mathrm{p}$ na análise bruta. A qualidade do ajuste do modelo foi verificada por 
meio do teste Hosmer e Lemeshow. Os dados foram analisados no software The Statistical Package for the Social Sciences (SPSS), versão 20.0, com nível de significância estabelecido de $5 \%$.

\section{Resultados}

Devido às barreiras encontradas durante a coleta de dados e ao preenchimento incorreto de alguns questionários obteve-se um número de 2.885 participantes, dos quais 368 foram excluídos das análises por estarem fora da faixa etária estipulada (14 a 19 anos), resultando em uma amostra final de 2.517 adolescentes. A maioria dos participantes era do sexo feminino (56,1\%), de 17 a 19 anos, cursava o primeiro ano do ensino médio, estudava no turno diurno, era de família com renda mensal de até dois salários mínimos, filhos de mães com menos de oito anos de estudos e não trabalhava (Tabela 1).

TABELA 1 - Características sociodemográficas dos adolescentes Amazonenses, na amostra total e estratificada por sexo. Amazonas, $2011(\mathrm{n}=2.517)$.

\begin{tabular}{|c|c|c|c|}
\hline \multirow{3}{*}{ Variáveis } & \multirow{2}{*}{ Total } & \multicolumn{2}{|r|}{ Sexo } \\
\hline & & Masculino & Feminino \\
\hline & $n(\%)$ & $n(\%)$ & n (\%) \\
\hline \multicolumn{4}{|l|}{ Faixa etária (anos) } \\
\hline $14-16$ & $1.210(48,1)$ & $512(46,3)$ & $698(49,5)$ \\
\hline $17-19$ & $1.307(51,9)$ & $594(53,7)$ & $713(50,5)$ \\
\hline \multicolumn{4}{|l|}{ Ano escolar (ensino médio) } \\
\hline Primeiro & $1.103(43,8)$ & $516(46,7)$ & $587(41,6)$ \\
\hline Segundo & $895(35,6)$ & $264(32,9)$ & $531(37,6)$ \\
\hline Terceiro & $519(20,6)$ & $226(20,4)$ & $293(20,8)$ \\
\hline \multicolumn{4}{|l|}{ Turno } \\
\hline Diurno & $2.040(81,0)$ & $817(79,2)$ & $1.164(82,5)$ \\
\hline Noturno & $477(19,0)$ & $230(20,8)$ & $247(17,5)$ \\
\hline \multicolumn{4}{|l|}{ Renda familiar (salários) } \\
\hline Até dois & $1.612(64,0)$ & $662(59,9)$ & $950(67,3)$ \\
\hline de três a cinco & $710(28,2)$ & $333(30,1)$ & $377(26,7)$ \\
\hline seis ou mais & $195(7,8)$ & $111(10,0)$ & $84(6,0)$ \\
\hline \multicolumn{4}{|l|}{ Escolaridade materna } \\
\hline$<$ oito anos de estudo & $1.468(65,4)$ & $661(67,4)$ & $807(63,9)$ \\
\hline$\geq$ oito anos de estudo & $775(34,6)$ & $319(32,6)$ & $456(36,1)$ \\
\hline \multicolumn{4}{|l|}{ Situação ocupacional } \\
\hline Não trabalha & $2.061(81,9)$ & $854(77,2)$ & $1.207(85,5)$ \\
\hline Trabalha & $456(18,1)$ & $252(22,8)$ & $204(14,5)$ \\
\hline
\end{tabular}

n: frequência absoluta; \%: frequência relativa.

Considerando as prevalências da AFMV suficiente na amostra total e de acordo com as variáveis independentes, foram observadas diferenças entre os critérios em todas as categorias das variáveis analisadas. Porém, a concordância entre as prevalências de AFMV suficiente entre os dois critérios, na amostra total, foi moderada, com índice kappa igual a 0,58, concordando em 91,5\% dos casos (Tabela 2).

$\mathrm{Na}$ análise bivariada para a identificação dos fatores associados, as variáveis sexo e faixa etária se associaram com a AFMV suficiente no critério de pelo menos 300 minutos/semana, enquanto que para o critério de pelo menos 420 minutos/sema- 
TABELA 2 - Prevalências de atividade física moderada/vigorosa na amostra total e de acordo com as variáveis independentes investigadas. Amazonas, 2011 ( $n=2.517)$.

\begin{tabular}{|c|c|c|}
\hline \multirow{2}{*}{ Variáveis } & 300 minutos/semana & 420 minutos/semana \\
\hline & $\%(I C 95 \%)$ & $\%(I C 95 \%)$ \\
\hline Amostra total & $15,5(14,2-16,7)^{*}$ & $7,0(6,0-7,0)^{*}$ \\
\hline \multicolumn{3}{|l|}{ Sexo } \\
\hline Masculino & $23,2(21,6-24,6)$ & $10,3(9,1-11,3)$ \\
\hline Feminino & $9,5(8,4-10,5)$ & $4,3(3,5-5,0)$ \\
\hline \multicolumn{3}{|l|}{ Faixa etária (anos) } \\
\hline $14-16$ & $17,1(15,7-18,3)$ & $7,2(6,2-8,1)$ \\
\hline $17-19$ & $14,1(12,7-15,2)$ & $6,7(5,7-7,5)$ \\
\hline \multicolumn{3}{|l|}{ Ano escolar (ensino médio) } \\
\hline Primeiro & $17,1(15,7-18,3)$ & $8,1(7,0-9,0)$ \\
\hline Segundo & $14,4(13,1-15,5)$ & $5,5(4,6-6,3)$ \\
\hline Terceiro & $14,1(12,7-15,3)$ & $7,1(6,1-7,9)$ \\
\hline \multicolumn{3}{|l|}{ Turno } \\
\hline Diurno & $15,1(13,7-16,3)$ & $6,6(5,7-7,4)$ \\
\hline Noturno & $17,2(15,7-18,4)$ & $8,6(7,5-9,5)$ \\
\hline \multicolumn{3}{|l|}{ Renda familiar (salários) } \\
\hline Até dois & $14,3(13,0-15,5)$ & $6,2(5,3-7,0)$ \\
\hline de três a cinco & $17,3(15,8-18,5)$ & $7,0(6,0-7,8)$ \\
\hline seis ou mais & $19,5(17,9-20,8)$ & $6,2(5,3-7,0)$ \\
\hline \multicolumn{3}{|l|}{ Escolaridade materna } \\
\hline$<$ oito anos de estudo & $15,7(14,3-16,9)$ & $7,8(6,8-8,7)$ \\
\hline$\geq$ oito anos de estudo & $14,1(12,7-15,2)$ & $5,0(4,2-5,7)$ \\
\hline \multicolumn{3}{|l|}{ Situação ocupacional } \\
\hline Não trabalha & $15,8(14,4-17,0)$ & $7,3(6,3-8,2)$ \\
\hline Trabalha & $14,5(13,2-15,7)$ & $5,5(4,6-6,3)$ \\
\hline
\end{tabular}

\%: frequência relativa; IC95\%: intervalo de confiança de 95\%. *kappa = 0,58; (IC95\% = 0,53-0,63); p $<0,001$.

na foram observadas associações com o sexo, ano escolar e escolaridade materna.

$\mathrm{Na}$ análise múltipla observou-se no critério de pelo menos 300 minutos/semana que os adolescentes do sexo masculino ( $\mathrm{OR}=3,02$; IC95\%: 2,36-3,86) e os mais novos (14-16 anos) $(\mathrm{OR}=1,38$; IC95\%: 1,04-1,84) e aqueles que não trabalhavam $(\mathrm{OR}=1,42$; IC95\%: 1,01-1,98) tiveram mais chances de serem ativos fisicamente quando comparados, respectivamente, aos seus pares do sexo feminino, mais velhos (17 a 19 anos) e que trabalhavam. O modelo final apresentou boa qualidade de ajuste de acordo com o teste de Hosmer e Lemeshow $\left(X^{2}=4,22 ; \mathrm{p}=0,837\right)$. Em relação ao critério de pelo menos 420 minutos/semana foi observado que os adolescentes do sexo masculino ( $\mathrm{OR}=2,45$; IC95\%: 1,71-3,41) e aqueles que não trabalhavam $(\mathrm{OR}=2,10$; IC95\%: 1,23-3,58) apresentaram mais chances de serem ativos fisicamente quando comparados às adolescentes do sexo feminino e aos que trabalhavam, respectivamente. O teste de Hosmer e Lemeshow indicou bom ajuste do modelo $\left(X^{2}=4,53 ; \mathrm{p}=0,806\right)$ (Tabela 3$)$. 
TABELA 3 - Análise de associação entre a atividade física moderada/vigorosa, classificada de acordo com os dois critérios (300 e 420 minutos/semana), e as variáveis independentes investigadas. Amazonas, $2011(n=2.517)$.

\begin{tabular}{|c|c|c|c|c|}
\hline \multirow{2}{*}{ Variáveis } & \multicolumn{2}{|c|}{300 minutos/semana } & \multicolumn{2}{|c|}{420 minutos/semana } \\
\hline & OR (IC95\%) & OR* (IC95\%) & OR (IC95\%) & $\mathrm{OR}^{*}(\mathrm{IC} 95 \%)$ \\
\hline \multicolumn{5}{|l|}{ Sexo } \\
\hline Masculino & $2,89(2,30-3,62)$ & $3,02(2,36-3,86)$ & $2,54(1,84-3,51)$ & $2,45(1,71-3,41)$ \\
\hline Feminino & 1 & 1 & 1 & 1 \\
\hline \multicolumn{5}{|l|}{ Faixa etária (anos) } \\
\hline $14-16$ & $1,26(1,02-1,56)$ & $1,38(1,04-1,84)$ & $1,07(0,79-1,46)$ & $1,19(0,79-1,79)$ \\
\hline $17-19$ & 1 & 1 & 1 & 1 \\
\hline \multicolumn{5}{|l|}{ Ano escolar (ensino médio) } \\
\hline Terceiro & $0,79(0,59-1,06)$ & $1,13(0,77-1,64)$ & $0,86(0,59-1,30)$ & $1,26(0,76-2,09)$ \\
\hline Segundo & $0,81(0,64-1,04)$ & $0,96(0,73-1,26)$ & $0,66(0,46-0,95)$ & $0,71(0,47-1,07)$ \\
\hline Primeiro & 1 & 1 & 1 & 1 \\
\hline \multicolumn{5}{|l|}{ Turno } \\
\hline Diurno & $0,86(0,66-1,12)$ & $0,80(0,59-1,09)$ & $1,34(0,93-1,93)$ & $0,75(0,49-1,15)$ \\
\hline Noturno & 1 & 1 & 1 & 1 \\
\hline \multicolumn{5}{|l|}{ Renda familiar (salários) } \\
\hline Até dois & 1 & 1 & 1 & 1 \\
\hline de três a cinco & $1,26(0,99-1,60)$ & $1,17(0,88-1,55)$ & $1,20(0,86-1,67)$ & $1,23(0,84-1,82)$ \\
\hline seis ou mais & $1,45(0,99-2,13)$ & $1,37(0,87-2,14)$ & $0,92(0,50-1,71)$ & $0,87(0,41-1,86)$ \\
\hline \multicolumn{5}{|l|}{ Escolaridade materna } \\
\hline$<$ oito anos de estudo & $1,14(0,89-1,46)$ & $1,10(0,85-1,42)$ & $0,63(0,43-0,92)$ & $1,47(0,99-2,15)$ \\
\hline$\geq$ oito anos de estudo & 1 & 1 & 1 & 1 \\
\hline \multicolumn{5}{|l|}{ Situação ocupacional } \\
\hline Não trabalha & $1,11(0,83-1,47)$ & $1,42(1,01-1,98)$ & $0,74(0,48-1,14)$ & $2,10(1,23-3,58)$ \\
\hline Trabalha & 1 & 1 & 1 & 1 \\
\hline
\end{tabular}

OR: Odds Ratio; IC95\%: Intervalo de confiança de 95\%. *Análise ajustada por todas variáveis.

\section{Discussão}

Os principais resultados do presente estudo demostraram concordância moderada entre os critérios de pelo menos 300 e 420 minutos/semana para classificação dos níveis de AFMV, nos adolescentes do Amazonas. Os fatores associados foram semelhantes em ambos os critérios, com exceção da faixa etária.

Quando comparadas as prevalências de AFMV suficiente entre os critérios, na amostra total, 214 adolescentes a mais atenderam às recomendações de AF classificada de acordo com o critério de 300 minutos/semana em comparação à quando empregados os 420 minutos/semana. Ainda, foram observadas diferenças significativas entre as prevalências de AFMV em todos os subgrupos de adolescentes, o que demonstra que ao classificá-los como fisicamente ativos no critério de 300 minutos/semana, esses adolescentes podem presumir que terão os mesmos benefícios daqueles que cumpriram os 420 minutos/semana.

Entretanto, em estudo prévio, Martinez-Gomes et al. ${ }^{19}$ verificaram que um volume de $A F M V \geq 385$ minutos/semana discriminava adolescentes com peso saudável daqueles com excesso de peso, enquanto um volume de AFMV $\geq 343$ minutos/semana discriminava aqueles com quantidades saudáveis de gordura corporal daqueles com gordura em excesso. Outros pesquisadores sugeriram pontos de corte de AFMV similares ( $\geq 320$ minutos/semana) para discriminar pro- 
blemas com excesso de peso em adolescentes ${ }^{20-21}$. Dessa forma, supõe-se que ao adotar como critério os 300 ao invés de 420 minutos/semana, os esforços para o desenvolvimento de políticas públicas em saúde destinadas a promoção da AF podem ser reduzidos, uma vez que as prevalências dos inquéritos que utilizarem esse critério podem estar superestimadas. Assim sendo, se o critério de 420 minutos/ semana foi estabelecido com base nos benefícios para a saúde dos adolescentes é importante que o mesmo seja utilizado, pois para reduzir riscos à saúde como, por exemplo, o excesso de peso, quantidades mínimas de AFMV são necessárias.

A respeito dos fatores associados, observou-se que, dentre os sete investigados, um (faixa etária) se associou apenas com a AFMV suficiente no critério de pelo menos 300 minutos/semana, dois (sexo e situação ocupacional) com a AFMV suficiente em ambos os critérios e quatro (turno, ano escolar, renda e escolaridade materna) não se associaram ao desfecho em nenhum dos critérios. Esses resultados indicam que, considerando-se os fatores em questão, a associação com a AFMV é semelhante e, portanto, acredita-se que quando o objetivo principal de um estudo for identificar os fatores sociodemográficos associados a esse desfecho, com exceção da faixa etária, ambos os critérios de classificação podem ser utilizados.

Cabe ressaltar que as particularidades metodológicas dos estudos prévios (como, por exemplo, as características da amostra investigada e os diferentes instrumentos utilizados para mensurar a $\mathrm{AF}^{3-10}$ ) podem resultar em associações distintas entre os achados dos mesmos ${ }^{5}$. No caso deste estudo, em que a faixa etária esteve associada à AFMV em apenas um dos critérios, pode-se supor que a associação com esse fator, especificamente, depende do critério adotado ou, ainda, que outros fatores possam contribuir para que quantidades mais elevadas de AFMV (como os 420 minutos/semana) sejam atingidas ou não, independentemente da faixa etária. É necessário que estudos futuros investiguem outros fatores (como biológicos, culturais e comportamentais, por exemplo) para verificar se sua relação com a AFMV depende do critério de classificação.

O presente estudo apresenta algumas limitações, dentre as quais destaca-se o uso de uma medida subjetiva de AF que pode ter resultado em sub ou superestimação das prevalências, em função do viés de memória. Os municípios incluídos apresentam características específicas relacionadas, por exemplo, à segurança púbica e infraestrutura, que podem ter influenciado nas prevalências dos níveis de AF e consequentemente nos fatores associados.

Apesar disso, destaca-se que o estudo apresenta pontos fortes, principalmente por ser um dos primeiros realizados com adolescentes da região Norte do Brasil, no estado do Amazonas, os quais foram selecionados a partir de uma amostragem probabilística e as amostras obtidas foram representativas dos municípios envolvidos, resultando em elevada validade externa. Ademais, os resultados encontrados poderão auxiliar os pesquisadores da área na tomada de decisão a respeito de qual critério adotar para classificação da AFMV no sentido de estarem cientes dos impactos e efeitos de cada critério para as estimativas de AF e os fatores associados.

Conclui-se que a concordância entre os critérios de classificação da AFMV de pelo menos 300 e 420 minutos/semana entre os adolescentes do Amazonas foi moderada e os fatores associados à AFMV suficiente foram semelhantes em ambos os critérios, exceto a faixa etária. Sugere-se que a escolha de um ou outro critério de classificação da AFMV seja realizada com cautela, considerando-se os objetivos do estudo. Acredita-se que em pesquisas que tenham por finalidade identificar, exclusivamente, os fatores associados à $\mathrm{AF}$ suficiente, o uso dos 300 minutos/semana é aceitável, devendo-se assumir, porém, que as prevalências do desfecho positivo 
podem ser superestimadas. Entretanto, sabendo-se que os fatores associados à $\mathrm{AF}$ são semelhantes em ambos os critérios e que quantidades mais elevadas de AFMV proporcionam maiores benefícios à saúde, recomenda-se o uso do critério de classificação da AFMV de pelo menos 420 minutos/semana, conforme proposta mais recente da Organização Mundial de Saúde.

\section{Colaboradores}

R. M. S. Puga Barbosa (0000-0003-1770-7777) e M. V. Nahas (0000-0002-43784031) contribuíram na elaboração do projeto de pesquisa e revisão crítica do manuscrito. A. A. Pinto (0000-0002-7931-3987), G. S. Claumann (0000-00023859-9106) e H. C. C. Angelo (0000-0002-2871-5724) participaram igualmente da redação e revisão crítica do manuscrito, realizaram a análise e interpretação dos dados. A. Pelegrini (0000-0001-8862-9636) idealizou o trabalho e participou da redação e revisão crítica do manuscrito.

\section{Conflito de interesses}

Os autores declaram não haver conflitos de interesses.

\section{Referências}

1. Rigoni PAG, Júnior JRA, Costa GNS, Vieira LF. Estágios de mudança de comportamento e percepção de barreiras para a prática de atividade física em universitários do curso de Educação Física. Rev Bras Ativ Fís e Saúde. 2012;17(2):87-92.

2. World Health Organization. Global recommendations on physical activity for health. Geneva: WHO; 2010. Acesso em 21 de setembro de 2016. Disponível em: http://apps. who.int/iris/bitstream/10665/44399/1/9789241599979_eng.pdf

3. Alberto AAD, Figueira Junior AJ, Miranda MLJ. Avaliação da atividade física na promoção da saúde de adolescentes brasileiros: revisão sistemática. Rev Bras Promoç Saúde. 2013;26(3):426-35.

4. Castro JAC, Nunes, HEG, Silva DAS. Prevalência de obesidade abdominal em adolescentes: associação entre fatores sociodemográficos e estilo de vida. Rev Paul Pediatr. 2016;34(3):343-51.

5. Coledan DHC, Ferraiol PF, Pires Junior R, Ribeiro EAG, Ferreira MAC, Oliveira AR. Concordância entre dois pontos de corte para atividade física e fatores associados em jovens. Rev Paul Pediatr. 2014;32(3):215-22.

6. Cureau FV, Duarte PM, Santos DLD, Reichert FF. Autopercepção de saúde em adolescentes: prevalência e associação com fatores de risco cardiovascular. Rev Bras Ativ Fís e Saúde. 2013;18(6):750-60.

7. Farias Júnior JC, Mendes JKF, Barbosa DBM, Lopes AS. Fatores de risco cardiovascular em adolescentes: prevalência e associação com fatores sociodemográficos. Rev Bras Epidemiol. 2011;14(1):50-62.

8. Fernandes RA, Christofaro DGD, Casonatto J, Kawaguti SS, Ronque ERV, Cardoso JR, et al. Associação transversal entre hábitos alimentares saudáveis e não saudáveis e atividade física de lazer em adolescentes. J Pediatr. 2011;87(3):252-6.

9. Farias Júnior JC, Lopes AS, Mota J, Hallal PC. Prática de atividade física e fatores associados em adolescentes no Nordeste do Brasil. Rev Saúde Pública. 2012;46(3):505-15.

10. Felden EPG, Filipin D, Barbosa DG, Andrade RD, Meyer C, Beltrame TS et al. Adolescents with excessive daytime sleepiness spend more time in sedentary behavior. Rev Bras Med Esporte. 2016;22(3):186-90.

11. Barufaldi LA, Abreu GA, Coutinho ESF, Bloch KV. Meta-analysis of the prevalence of physical inactivity among Brazilian adolescents. Cad Saúde Pública. 2012; 28(6):1019-32.

12. Dumith SC, Gigante DP, Domingues MR, Kolh HW. Physical activity change during adolescence: a systematic review and a pooled analysis. Int J Epidemiol. 2011;40(3):685-98.

13. Moraes AC, Guerra PH, Menezes PR. The worldwide prevalence of insufficient physical activity in adolescents; a systematic review. Nutr Hosp. 2013;28(3):575-84.

14. Lima DF, Levy RB, Luiz OC. Recomendações para atividade física e saúde: consensos, controvérsias e ambiguidades. Rev Panam Salud Pública. 2014;36(3):164-70. 
15. Luiz RR, Magnanini MMF. O tamanho da amostra em investigações epidemiológicas. Cad Saude Colet. 2000;8(2):9-28

16. Silva KS, Lopes AS, Hoefelmann LP, Cabral LG, De Bem MF, Barros MV, et al. Projeto Compac (Comportamento dos Adolescentes Catarinenses): aspectos metodológicos, operacionais e éticos. Rev Bras Cineantropom Desempenho Hum. 2013;15(1):1-15.

17. Cohen J. A coefficient of agreement nominal scales. Edu Psychol Meas. 1960;20(1):37-46.

18. Landis JR, Koch GG. The measurement of observer agreement for categorical data. Biometrics. 1977; 33(1):159-74.

19. Martinez-Gomez D, Ruiz JR, Ortega FB, Veiga OL, Moliner-Urdiales D, Mauro B, et al. Recommended levels of physical activity to avoid an excess of body fat in European adolescents: the HELENA Study. Am J Prev Med. 2010;39(3):203-11.

20. Laguna M, Ruiz JR, Lara MT, Aznar S. Recommended levels of physical activity to avoid adiposity in Spanish children. Pediatr Obes. 2013;8(1):69-9.

21. Jiménez-Pavón D, Konstabel K, Bergman P, Ahrens W, Pohlbeln H, Hadjigeorgiou C, et al. Physical activity and clustered cardiovascular disease risk factors in young children: a cross-sectional study (the IDEFICS study). BMC Medicine. 2013;11(1):172.

Endereço para

Correspondência

Andreia Pelegrini

andreia.pelegrini@udesc.br
Centro de Ciências da Saúde e do Esporte,

Universidade do Estado de Santa Catarina.

Grupo de Estudos e Pesquisa em

Cineantropometria-GEPECIN

Rua Pascoal Simone, 358, Coqueiros,

Florianópolis - SC. CEP: 88080-350

Telefone: (48) 3664-8695
Recebido 04/10/2016

Revisado 29/12/2016

$18 / 02 / 2017$

$24 / 02 / 2017$

Aprovado 29/03/2017 